BULLETIN Bulletin hispanique

HISPANIQUE Université Michel de Montaigne Bordeaux

116-1 | 2014

Varia

\title{
Le paysage militaire et le « suave mari magno » de Lucrèce dans les comedias caldéroniennes
}

\section{Marie-Eugénie Kaufmant}

\section{(2) OpenEdition}

1 Journals

Édition électronique

URL : http://journals.openedition.org/bulletinhispanique/3108

DOI : 10.4000/bulletinhispanique.3108

ISSN : 1775-3821

Éditeur

Presses universitaires de Bordeaux

\section{Édition imprimée}

Date de publication : 1 juin 2014

Pagination : 141-167

ISBN : 978-2-86781-931-5

ISSN : 0007-4640

Référence électronique

Marie-Eugénie Kaufmant, «Le paysage militaire et le « suave mari magno » de Lucrèce dans les comedias caldéroniennes », Bulletin hispanique [En ligne], 116-1 | 2014, mis en ligne le 01 juin 2017, consulté le 24 mai 2020. URL : http://journals.openedition.org/bulletinhispanique/3108 ; DOI : https:// doi.org/10.4000/bulletinhispanique.3108 


\title{
Le paysage militaire et le "suave mari magno" de Lucrèce dans les comedias caldéroniennes
}

\author{
Marie-Eugénie Kaufmant \\ Université de Bretagne Occidentale
}

Dans les premières comedias de Calderón de la Barca, la représentation scénique restreinte de la guerre délivre une leçon sur l'identification tragique à travers la fascination pour le paysage militaire, la théatralisation du "suave mari magno " de Lucrèce et la téichoscopie.

Mots-clés : Calderón de la Barca, comedias, guerre, Lucrèce, tragique, téichoscopie.

En las primeras comedias de Calderón de la Barca, la representación escénica restringida de la guerra desemboca en una lección sobre la identificación trágica, a través de la fascinación por el paisaje militar, la teatralización del "suave mari magno " de Lucrecio y la ticoscopia.

Palabras claves: Calderón de la Barca, comedias, guerra, Lucrecio, trágico, ticoscopia.

In Calderón de la Barca's first comedias, the restricted staging of war leads to a lesson on tragic identification, through the fascination for the military landscape, the dramatization of Lucretius" "suave mari magno " and teichoscopie.

Keywords : Calderón de la Barca, comedias, war, Lucretius, tragic, teichoscopy.

$\mathrm{P}$ edro Calderón de la Barca semble avoir été fasciné par la représentation de la guerre dans ses premières comedias. Cinq au moins des pièces de la Primera parte publiée en $1636^{1}$ représentent un fait militaire : la plus connue

1. Édition utilisée des œuvres citées de cette Primera parte, à l'exception de La vida es sueño, El príncipe constante et Amar después de la muerte: Pedro Calderón de la Barca, Comedias, I: Primera parte de Comedias, Madrid, Biblioteca Castro, 2006.

Bulletin Hispanique, Tome 116, n 1 - juin 2014 - p. 141-167. 
est La vida es sueño ${ }^{2}$ où le troisième acte résonne des échos de la guerre civile qui se déchaîne entre les partisans de Segismundo et ceux du Roi Basilio. Parmi les pièces qui mettent en scène des conflits armés légendaires, il y a aussi La puente de Mantible ${ }^{3}$, drame épico-légendaire de thème chevaleresque inspiré par les légendes de Charlemagne. Cette pièce fut représentée au palais en 1630 et sans doute composée peu avant ${ }^{4}$. La gran Cenobia ${ }^{5}$, quant à elle, s'organise autour de la guerre légendaire que se livrent l'empereur romain Aurélien (Aureliano) et la reine d'Asie Zénobie (Cenobia). Cette pièce serait une des plus précoces de Calderón : une représentation eut lieu au palais en 1625, mais on ne peut affirmer que ce soit une première. Le même intérêt pour la chose militaire caractérise El principe constante probablement composée fin 1628. Ce drame religieux inclassable dramatise le martyre de Fernando relaté par des chroniques portugaises du XV siècle : en 1437 , le roi don Duarte du Portugal part à la conquête de Tanger. L'expédition militaire représentée à l'acte I est un désastre. L'infant don Fernando est laissé en otage et meurt en captivité. À l'acte III, Alfonso $\mathrm{V}$, héritier du trône portugais, mène une nouvelle expédition contre les armées maures de Fez et rachète ainsi la dépouille du martyr don Fernando qui, dans le drame, éclaire le combat de son aura sacrée. La symbolique de la guerre, dans cette pièce qui a pour source un fait historique, est soumise à la vision métaphysique qui entoure la figure légendaire de don Fernando ${ }^{6}$.

Deux autres pièces de Calderón consacrent, quant à elles, l'intégralité de leur intrigue à la dramatisation de faits armés qui sont bien plus proches de la réception de l'époque : vraisemblablement représentée pour la première fois en 1628 à l'occasion de la venue de Spinola à Madrid, El sitio de Bredá ${ }^{7}$ met en scène un évènement historique immédiat dans la mémoire du public, le siège stratégique mené par le Marquis de Spinola contre la ville de Breda, fief de la maison d'Orange en 1625 . Une autre pièce qui forme ce corpus de pièces de Calderón caractérisées par la représentation du fait guerrier est El Tuzani de la Alpujarra ${ }^{8}$, pièce plus connue sous le nom de Amar después de la muerte qui ne fait pas partie des pièces de la Primera parte, mais qui s'en rapproche par sa date de composition cependant incertaine ${ }^{9}$ : elle met en scène la répression militaire

2. Édition utilisée : Pedro Calderón de la Barca, La vida es sueño, Madrid, Castalia, 1994.

3. Comedias, I, op. cit., pp. 483-573.

4. D'après l'introduction à l'édition Castro.

5. Ibid., pp. 307-396.

6. Pour cette pièce, je n'utilise pas la version imprimée de la Primera parte, mais celle du manuscrit de la BNM. Cette version est celle éditée dans Pedro Calderón de la Barca, El príncipe constante, Madrid, Cátedra, 1996.

7. Comedias, I, op. cit., pp. 953-1052.

8. Il existe plusieurs versions assez proches de cette pièce avec une ambivalence quant au titre, notamment la suivante : Pedro Calderón de la Barca, El Tuzaní de la Alpujarra, Sevilla, Guadalmena, 1998. Pour ma part, j'utiliserai l'édition critique de Erik Coenen : Pedro Calderón de la Barca, Amar después de la muerte, Madrid, Catedra, 2008.

9. Erik Coenen, dans son introduction à l'édition critique de l'œuvre, remet en cause cette date bien qu'il reconnaisse que son style l'associe à la première période de Calderón. Voir l'introduction à $O p$. cit., pp. 47-48. 
contre la rébellion morisque des Alpujarras et se concentre sur le siège de Galera, violemment conquise et dévastée par les armées de don Juan d'Autriche en 1570. Il s'agit d'une pièce où Calderón prend quelques libertés poétiques quant à la réalité historique et géographique et où il semble avant tout adopter une attitude critique vis-à-vis du pouvoir répressif contre les Morisques, comme l'ont souligné plusieurs critiques ${ }^{10}$. Hannaá Walzer est moins catégorique quant à cet aspect critique qui soulignerait surtout l'autenticité de la Foi catholique comme véritable critère d'évaluation positive par une double discrimination des faux convertis et des mauvais vieux chrétiens ${ }^{11}$. Erik Coenen, quant à lui, considère que Calderón transcende cette vision particulière historique au service d'une valeur universelle de la pièce qui délivrerait une caractérisation négative de la guerre et des sièges militaires en général ${ }^{12}$. David García Hernán ${ }^{13}$ pour sa part, analyse subtilement la «culture de la guerre». Loin de n'être que le reflet d'une propagande imposée, elle témoigne d'une certaine conscience critique et propose une vision bienveillante de l'Autre propre à satisfaire le goût du public mêlé du corral ${ }^{14}$. Au-delà d'une conscience critique, Christophe Couderc ${ }^{15}$ suggère à ce sujet la nécessité de "vérité poétique " à travers la représentation nuancée de la guerre, que Amar después de la muerte semble dépasser par le pathétique du point de vue morisque. À partir de cette pièce précisément, et en la comparant à d'autres, il apparaît que se fait jour une remarquable conscience de la théâtralité de la guerre.

La production de ces comedias est effectivement conçue dans la tradition représentative du corral auquel elles étaient en majorité destinées vers 1630, même si la première trace que l'on conserve de certaines d'entre elles est une représentation au palais. Amar después de la muerte, bien que composée légèrement après, n'échappe pas à cette règle. Le texte dramatique de ces pièces renvoie à une réalité scénique sobre et limitée qui est celle du corral de comedias, bien mise en lumière par Anne Teulade lorsqu'elle étudie l'espace dans El sitio de Bredá ${ }^{16}$.

10. Voir articles de José Alcalá Zamora, "Individuo e historia en la estructura teatral de $E l$ Tuzani de la Alpujarra", in Calderón. Actas del "Congreso internacional sobre Calderón y el teatro español del Siglo de Oro" (Madrid, 8-13 de junio de 1981), I, Madrid, CSIC, 1983, pp. 343-363 et de José Miguel Caso González, "Calderón y los Moriscos de las Alpujarras », in id., pp. 393-402.

11. Hannaá Walzer, "Los moriscos de Amar después de la muerte ", Ayer y hoy de Calderón, Madrid, Castalia, 2002, pp.133-145.

12. Voir Erik Coenen, "Calderón y la guerra : del sitio de Bredá al sitio de Galera ", Nueva revista de filología hispánica, vol. LVI, Nº 1, México, Colegio de México, Février-Juillet 2008, pp. 31-51.

13. David García Hernán, La cultura de la guerra y el teatro del Siglo de Oro, Madrid, Silex, 2006. [NDLR : Voir, dans ce même numéro, son article, «Los gustos del público y la cultura de la guerra en los géneros literarios de ficción del Siglo de Oro español ».]

14. La cultura, chapitre IV.4 : "La presencia de las ideas de "los otros" ", pp. 205-216.

15. Christophe Couderc, "David García Hernán, La cultura de la guerra y el teatro del Siglo de Oro ", Mélanges de la Casa de Velázquez, 38-1, 2008, pp. 307-309 [en ligne]

http://mcv.revues.org/1116

16. Anne Teulade, «El espacio plural en El sitio de Bredá de Calderón : lo visible y lo invisible 
Le propos de cette étude est donc d'envisager, du point de vue de l'esthétique théâtrale, et à travers la représentation dramatique de l'espace guerrier, les traits saillants de la poétique caldéronienne de la guerre. Bien que lié au tragique, comme le démontre Benjamín Torrico à propos de ce qu’il considère dès la dramaturgie de Lope de Vega comme un veritable "sous-genre guerrier " ${ }^{17}$, le fait militaire ne semble pas être a priori un élément de taxinomie des comedias étant donné la variété des types de pièces concernées, des pièces historiques aux pièces légendaires en passant par les drames tragiques ou métaphysiques.

Afin de souligner la spécificité de la poétique théâtrale de la guerre, l'analyse s'organisera selon la dialectique qui met en relation la poétique symbolique avec les données de la représentation théâtrale particulière à ce théâtre. Pour ce faire, après un bref examen des possibilités significatives de mise en scène du fait guerrier dans ce théâtre, il s'agira de commenter les perspectives offertes sur le champ de bataille et le paysage militaire à travers le décor verbal pour analyser ensuite l'influence du «suave mari magno » de Lucrèce et la symbolique théâtrale des téichoscopies ou " regards depuis la muraille ", très fréquents dans le théâtre caldéronien.

\section{Mise en SCÈne De L'ESPACE DE GUERRE}

Pour les comedias de corral, la représentation de la guerre se heurte à un écueil, celui qui caractérise toute mise en scène des espaces extérieurs et ouverts dans ce type de pièces : la scène, en effet, peut difficilement figurer l'étendue spatiale et rendre le mouvement des batailles et des opérations militaires. La figuration de la guerre devient alors contextuelle et se fait par une série de conventions scéniques. Le contexte militaire est le plus souvent évoqué en horsscène. L'étendue du champ de bataille est suggérée par les voix-off du combat représenté comme espace invisible tel que l'a analysé Anne Teulade ${ }^{18}$. De fait, la contiguïté permanente du champ de bataille dans El sitio de Bredá est rendue par l'utilisation des conventions phoniques qui associent l'espace scénique à l'espace guerrier. Plus généralement dans l'ensemble des pièces concernées par la guerre, les didascalies externes multiplient les mentions de sons extrascéniques de clairons et de trompettes, de roulements de tambours, de bruits de tirs ou de cris de guerre : "Tocan un clarín", ou même "Tocan", "Disparan", "vanse tocando cajas ", " ruido de cajas ", "Dentro cajas ", "Tocan al arma", "Tocan clarines ", "Suena ruido de armas ", etc. Ces procédés phoniques sont répétitifs d'une pièce à l'autre et même à l'intérieur d'une même pièce. Par

en un drama histórico-épico ", in El Siglo de Oro en escena. Homenaje a Marc Vitse, Toulouse, PUM/ Consejería de Educación de la Embajada de Espańa en Francia, 2006, pp. 1011-1024. Voir p. 1012.

17. "subgénero bélico » selon Benjamín Torrico, « De sitios y sitiados : el subgénero bélico como nueva tragedia ", Hacia la tragedia áurea : lecturas para un nuevo milenio, Madrid, Université de Navarre/ Iberoamericana, 2008, pp. 277-285.

18. Art. cité. 
exemple, à l'acte III de La vida es sueño, les échos guerriers qui envahissent tout l'espace, y compris le palais, sont commentés par les habitants du palais et par Basilio, alors que résonnent depuis le hors-scène les roulements de tambours guerriers et les échos des vivats des partisans de Segismundo et de ceux de Basilio : "se oye resonar en lo profundo / de los montes, el eco repetido, / unos " ¡Astolfo ! " y otros " ¡Segismundo ! " ${ }^{19}$. Tout l'acte III de La vida es sueño est construit avec une toile de fond sonore qui illustre le contexte de guerre civile comme le soulignent les didascalies répétées qui évoquent la marche guerrière et les tirs : "tocan al arma ${ }^{20}$, "dentro cajas " ${ }^{21}$, "Suena ruido de armas ${ }^{22}$, "Disparan dentro " ${ }^{23}$.

Le contexte sonore guerrier est souvent renforcé par les didascalies internes au texte dramatique qui explicitent les voix-off. C'est un procédé que l'on retrouve, par exemple, dans El principe constante où les voix-off sont diffusées des deux côtés de la scène, signifiant ainsi, avec l'appui des commentaires de Fernando et Enrique, l'encerclement des troupes portugaises par les troupes maures de Tarudante, d'une part, et du roi de Fez, d'autre part :

\begin{tabular}{ll} 
& (Tocan una trompeta.) \\
FER. & Mas iqué trompeta es esta, \\
& que alegre turba y la región molesta? \\
& (Tocan cajas a otro lado.) \\
& Y por esotra parte, \\
& Cajas se escuchan, música de Marte. \\
& ¿Qué será? \\
& \multicolumn{1}{c}{ Aquellos ecos, } \\
ENR. $\quad$ & ejércitos de Fez y Marruecos \\
& son, porque Tarudante \\
& al rey de Fez socorre, y arrogante \\
& el rey con gente viene, \\
& en medio cada ejército nos tiene \\
& de modo que cercados \\
& somos los sitiadores y sitiados. ${ }^{24}$
\end{tabular}

Ainsi, si la plupart du temps la bataille n'est pas représentée dans son étendue, elle est suggérée phoniquement comme contexte immédiat en vertu du principe de continuité qui est établi entre la scène et le hors-scène. Cela permet, dans ce dernier exemple, avec l'appui du décor verbal, de suggérer l'étendue spatiale stratégique du champ de bataille.

Il arrive cependant, dans de rares cas, que la bataille soit représentée par des scènes successives ou simultanées de combats individuels, où le mouvement d'entrée et de sortie de scène, répété, traduit l'espace itinérant à travers le champ

19. La vida es sueño, op. cit., v. 2437-2439.

20. Ibid., didascalies qui suivent les vers 2427,2491 et 3135 .

21. Ibid., didascalie qui suit le vers 3031.

22. Ibid., didascalie qui suit le vers 3059

23. Ibid., didascalie qui suit le vers 3070 .

24. El principe constante, op. cit., v. 827-831 et 834-841 
de bataille. Les didascalies de El principe constante illustrent effectivement une volonté de représentation métonymique de la bataille par la mise en scène d'une partie des combats individuels majeurs. Le combat commence par la domination chrétienne face aux troupes conduites par le capitaine maure Muley : "Salen DON ENRIQUE y DON JUAN retirando a los moros, y CUTINOO acuchillados por detrás ${ }^{25}$. L'espace de la fuite est rendu visible sur scène par les entrées et sorties de scène d'un groupe de soldats maures : "Huyen los moros, entran por una puerta y salen por otra ${ }^{26}$. Les capacités de la scène de corral sont exploitées à travers l'utilisation des deux portes qui viennent symboliser le parcours à travers l'espace itinérant du champ de bataille représenté sur scène, selon la convention de mobilité spatiale étudiée par Javier Rubiera Fernández ${ }^{27}$. Du reste, le décor verbal mis en œuvre par les personnages souligne l'effet de perspective :

\begin{tabular}{cl} 
ENR. & $\begin{array}{l}\text { Llenos de despojos quedan } \\
\text { de caballos y jinetes } \\
\text { estos campos. }\end{array}$ \\
CUT. & \multicolumn{1}{c}{ Mi señor, } \\
D. JU. & $\begin{array}{l}\text { idónde está, que no parece? } \\
\text { Tanto se empeñó en los moros } \\
\text { que ya de vista se pierde. }\end{array}$
\end{tabular}

Ainsi l'étendue du champ de bataille et de la domination chrétienne estelle créée en hors-scène par le décor verbal des personnages qui deviennent des spectateurs intermédiaires, comme il arrive souvent dans la comedia. Après l'arrivée des renforts, le combat se poursuit par la mise en scène des valets et la destinée guerrière des camps maures et chrétiens s'inverse visiblement à travers la représentation métonymique des combats individuels : "Sale CUTIÑO retirándose de ZELÍN y otros dos moros " ${ }^{29}$. Plus loin, le combat se recentre sur les couples des maîtres : "Salen peleando DON FERNANDO y el REY MORO, DON JUAN y otro moro. MULEY y DON ENRIQUE dan al tablado y éntranse, y salgan luego DON FERNANDO, retirándose del REY y de MULEY ${ }^{30}$. Le caractère schématique de ces mises en scène métonymiques de la bataille théâtralise non seulement la représentation stratégique de la bataille mais également les revirements de fortune des différents camps chrétiens et maures. Lorsqu'elle est représentée scéniquement, la bataille l'est par des combats individuels qui figurent les combats collectifs et soulignent la théâtralité de la guerre comme image de la fortune changeante.

25. Ibid., didascalie qui suit le vers 566 .

26. Ibid., didascalie qui suit le vers 572 .

27. Voir, au sujet de la convention de mobilité scénique et de l'espace itinérant scénique, Javier Rubiera Fernández, La construcción del espacio en la comedia española del Siglo de Oro, Madrid, Arco/Libros, 2005, pp. 99-124.

28. El príncipe constante, op. cit., v. 575-580.

29. Ibid., didascalie qui suit le vers 861 .

30. Ibid., didascalie qui suit le vers 889 . 


\section{LA PERSPECTIVE SUR LE CHAMP DE BATAILLE ET LE PAYSAGE MILITAIRE}

Tout l'art du décor verbal est de rendre visible l'espace guerrier à l'imagination du spectateur. Parfois même, le caractère invisible de l'espace de guerre en horsscène est théâtralisé selon la signification symbolique de la pièce. Au troisième acte de La vida es sueño, lorsque le soldat incite Segismundo à la sédition en lui montrant dans le monte l'étendue des troupes de partisans qui sont prêtes à le suivre, la perspective ouverte sur l'espace de guerre que sont les montagnes fait fonctionner la continuité entre l'espace scénique, et notamment le monte ou escalier scénique qui représente l'espace dramatique du monte, et les espaces en hors-scène : «Si piensas que te engañamos, / vuelve a ese monte soberbio / los ojos, para que veas / la gente que aguarda en ellos/ para obedecerte ${ }^{31}$. Le pronom personnel « ellos » qui reprend au pluriel monte crée linguistiquement la continuité entre la scène (et particulièrement le monte scénique désigné par le déictique " ese ") et la perspective en hors-scène qui doit permettre au public de s'imaginer l'étendue des troupes. Le caractère invisible de cet espace guerrier est théâtralisé à partir de la métaphore maîtresse de l'œuvre selon laquelle toute gloire humaine est songe : "Ya / otra vez vi aquesto mesmo / tan clara y distintamente / como agora lo estoy viendo, / y fue sueño $~_{32}$. La réplique de Segismundo souligne l'évanescence de la perspective théâtrale créée sur l'espace de guerre en hors-scène en la reliant à la thématique centrale qui anime l'expérience du héros.

Légion sont les perspectives ouvertes sur le champ de bataille, parfois même depuis les espaces intérieurs du palais depuis lequel est proférée la menace de guerre. L'espace de guerre semble toujours être conçu dans ces comedias comme un paysage immédiat. Au troisième acte de El principe constante qui multiplie ces perspectives vers l'espace du combat guerrier, alors que le roi maure et le roi Alfonso sont réunis au palais pour négocier le rachat de don Fernando, le paysage militaire est créé par la menace guerrière de don Alfonso selon des procédés d'anamorphose traditionnels pour évoquer le champ de bataille, où le combat prend la forme d'un paysage :

\section{[...] aquestas campañas llenas de sangre, de suerte que cuando el sol se levante halle los matices verdes esmeraldas y los pierda rubies cuando se acueste. ${ }^{33}$}

La préciosité des images relatives au paysage militaire envisagé souligne le chromatisme contrasté tandis que le déictique construit dans l'imagination du spectateur le paysage militaire dans le prolongement de la scène. La poétique

31. La vida es sueño, op. cit., v. 2344-2348.

32. Ibid., v. 2348-2351.

33. El principe constante, op. cit., v. 2165-2170. 
sanguinaire hyperbolique qui anime les menaces de guerre, dans des pièces comme El principe constante, crée un paysage où l'anamorphose entre l'espace ensanglanté du combat et la nature se caractérise par les métaphores d'un paysage printanier, notamment celles des pierres précieuses. Dans La puente de Mantible notamment, Fierabrás prononce à l'encontre de son rival une menace de guerre paysagée très développée :

$$
\begin{aligned}
& \text { [...] pienso en su demanda } \\
& \text { hacer que este valle hermoso } \\
& \text { con los cadáveres sea } \\
& \text { un bárbaro promontorio, } \\
& \text { tanto que el sol al nacer, } \\
& \text { viendo monte el que era soto, } \\
& \text { piense que ha errado el camino } \\
& \text { de sus celestiales tornos; } \\
& \text { las flores se han de mirar } \\
& \text { en los humanos arroyos } \\
& \text { de sangre y estos humildes } \\
& \text { céspedes que piso y toco, } \\
& \text { compitiendo los claveles, } \\
& \text { tendrán desdichas a logro, } \\
& \text { pues, a pesar del aurora, } \\
& \text { que con lágrimas y soplos } \\
& \text { quiso que naciesen verdes, } \\
& \text { querré yo que mueran rojos. }{ }^{34}
\end{aligned}
$$

La poétique hyperbolique du paysage guerrier qui se profile dans la menace du combat illustre la superbe démentielle de Fierabrás, capable comme un géant d'ériger une montagne de cadavres, métamorphosée en printemps sanguinaire où les ruisseaux de sang rivalisent avec la couleur des fleurs. Le paysage guerrier du monte devient symbolique de l'orgueil démesuré qui conduira Fierabrás à sa chute. Voici donc comment l'ultimatum ouvre des perspectives sur un paysage hyperboliquement métamorphosé tandis que les combats en tant que tels sont éludés ou réduits à des mises en scène schématiques. Parallèlement, à la fin de l'acte II, dans son inquiétude amoureuse, Floripes imagine le sort guerrier de son bien-aimé Guido blessé :

No podréis decirme todos
más de lo que yo me sé,
porque ya le he visto, ya,
dentro de mí misma hacer
piélagos de undosa sangre,
siendo su acero el desdén
del noto cuando sacude
las espigas de una mies.
Aqui derriba, alli mata
y son ruinas de sus pies
las victorias de sus manos.
Ya desmayado se ve,
despedazando el escudo,
mal guarnecido el arnés;

34. La puente de Mantible, op. cit., pp. 499-500. 


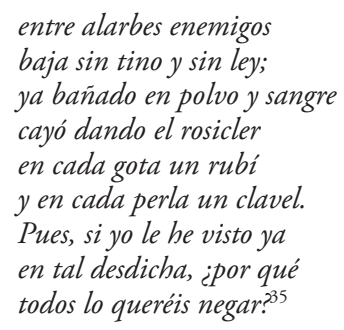

Dans l'imagination de l'amante comme dans celle du spectateur, le procédé de mise en perspective intériorisé est le même : à travers la puissance déictique de ces vers, le spectateur ne peut qu'entrevoir le paysage militaire où se débat Guido. La vision intériorisée du combat s'ouvre en une poétique paysagée écarlate qui nourrit en hors-scène l'imagination militaire du spectateur. L'insistance sur le regard imaginaire de Floripes, "yo le he visto ya », souligne la théâtralité langagière de ces représentations de l'espace militaire dans une sorte de mise en abyme du processus selon lequel le théâtre magnifie l'irreprésentable fait guerrier : sa violence indicible est pourtant dite à travers l'émotion du spectateur intérieur. Il s'agit d'une rhétorique de l'evidentia qui utilise la puissance théâtrale d'un langage destiné à " donner à voir " comme une évidence le spectacle imaginaire du combat à travers les hyperboles anamorphiques, océans de sang et paysages champêtres couverts d'une récolte de rouges œillets et de rubis dont le reflet est diffracté par la rosée.

Anne Teulade définit l'espace de guerre dans El sitio de Bredá comme un espace pluriel constitué par le décor verbal et par une " ampliation imaginaire de ce qui est matériellement perceptible par le spectateur $»^{36}$. Elle constate que la pièce élude paradoxalement la mise en scène des épisodes de batailles pour soumettre leur représentation à l'exaltation d'un spectacle militaire à travers le décor verbal. C'est le fait de nombreuses pièces caldéroniennes de la même période qui, faute de pouvoir représenter l'étendue spatiale de la bataille, exaltent la valeur paysagère et spectatoriale de la représentation guerrière verbale. Les déploiements militaires et le champ de bataille font partie de ces représentations d'espaces ouverts qui, selon Emilio Orozco Díaz ${ }^{37}$, fascinent Calderón et offrent des possibilités presque picturales à l'appréhension d'un paysage. Les espaces de guerre sont préférentiellement les espaces naturels ouverts que sont la mer, la campagne ou le monte : la représentation du fait militaire s'imprègne chez Calderón des conceptions paysagères naissantes qui animent ces espaces ouverts ${ }^{38}$. Sans revenir sur les autres éléments de mise en scène du fait militaire dans El sitio de Bredá, étudiés par Anne Teulade, il est intéressant de

35. Ibid., pp. 542-543.

36. Art. cité, citation traduite p. 1013.

37. Emilio Orozco Díaz, « Sentido de continuidad espacial y desbordamiento expresivo en el teatro de Calderón. El soliloquio y el aparte ", Calderón..., op. cit., pp. 125-164.

38. Voir notamment l'ouvrage issu de ma thèse de doctorat: Poétique des espaces naturels dans la comedia nueva, Madrid, Casa de Velázquez, 2010, pp. 338-347. 
commenter la poétique paysagère qui est orchestrée autour du déploiement des troupes espagnoles selon un aperçu de Madame Flora, qui devient alors une spectatrice intermédiaire du panache des armées ennemies :

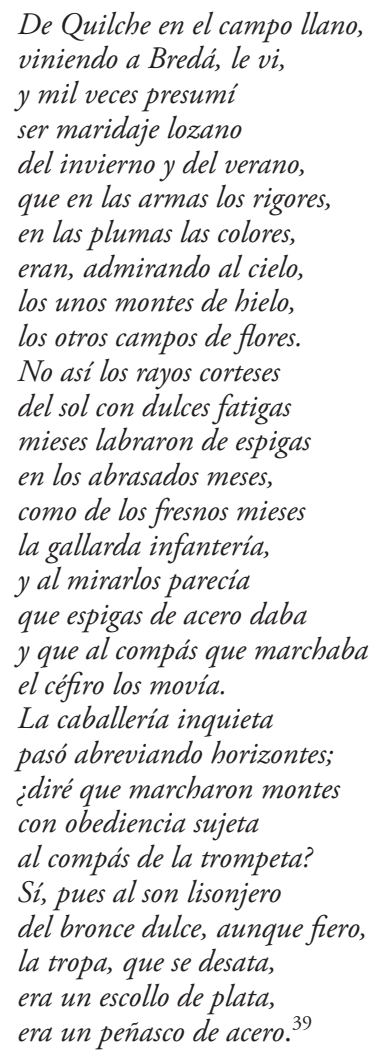

La description du déploiement des troupes espagnoles montre leur adéquation au paysage. Comme le souligne Anne Teulade, la représentation du fait militaire dans cette pièce privilégie, aux dépens d'une mise en scène des batailles, la démonstration d'une domination de l'espace par les Espagnols. L'hypotypose ou description en direct nourrit la rhétorique de l'evidentia qui donne à voir les troupes déployées dans l'espace comme un tableau paysagé. Il s'agit de l'aboutissement de la traditionnelle métaphore champêtre utilisée pour décrire l'étendue militaire. Dans cette tirade, le paysage militaire est décrit en plans successifs selon l'ordonnancement des troupes qui recrée les plans d'un paysage avec l'infanterie métaphorisée grâce à ses lances en champs de blés et la cavalerie qui se transforme en montagnes d'acier. Ces métaphores sont une justification de la victoire à venir puisque les champs de blés évoquent la récolte future et que la métaphore des montagnes véhicule un symbolisme d'invincibilité et d'abondance. 
On retrouve la même perspective sur le paysage de déploiement des troupes métaphorisé en paysage champêtre dans La puente de Mantible. Selon un phénomène de diffraction du point de vue (objectif et poétique), Irene souligne à son tour les métamorphoses que les armées de Floripes (fingiendo, imitando, parecen) imposent au paysage :

¿No ves
tus ejércitos marchando,
que a los dos vienen siguiendo,
montes de pluma fingiendo,
mares de acero imitando,
porque son, en tornasoles
en quien el sol se retrata,
las armas ondas de plata,
las plumas selvas de flores?
Las descogidas banderas,
que aves de viento parecen,
con colores desvanecen
los cielos por las esferas,
porque, dando al sol desmayos
con tornasoles sutiles,
le trasladan los abriles,
le tiranizan los mayos.
Vuelve los ojos y mira
tanto aplauso y pompa tanta
que el sol de verlos se espanta,
que el mar de oirlos se admira;
los montes, de sustentarlos,
deliran o se estremecen,
que montes vivos parecen
elefantes y caballos. ${ }^{40}$

L'invitation à la contemplation de la perspective militaire investit également le spectateur à travers la thématisation du regard et la sollicitation de l'ouïe. La métaphore champêtre se caractérise par les mêmes éléments du reflet et de la diffraction que dans la citation précédente où Floripes imagine son bien-aimé Guido en proie à la fureur du combat. La métaphore des miroitements irisés, " tornasoles ", permet la réfraction anamorphique des éléments de la nature à travers le prisme des armes et des drapeaux. Il s'agit d'une mise en perspective baroque de la traditionnelle métaphore caldéronienne de la compétition des éléments, qui nourrit la signification symbolique de l'œuvre selon laquelle Floripes est une figure de la démesure féminine. Le paysage militaire est dominé par les éléments du soleil, du monte et de la mer, soulignant ainsi l'intégration des troupes au paysage qui l'entoure et qui devient lui-même spectateur métaphorique d'un spectacle grandiose. Ainsi, dans ce théâtre de guerre, le spectateur est-il replacé par la rhétorique de l'evidentia au sein d'un cosmos dominé par la notion d'émulation guerrière où la démesure guerrière humaine introduit une notion de déséquilibre en condamnant les éléments eux-mêmes à la stupeur spectatoriale. 
Cependant, au-delà de cette fascination pour le paysage de guerre, un autre spectateur de la tradition antique semble servir de référence face au spectacle de la guerre : il s'agit du spectateur du "suave mari magno " de Lucrèce.

\section{LA THÉÂTRALISATION DU « SUAVE MARI MAGNO » DE LUCRÈCE}

Dans Amar después de la muerte, au cours du récit de la rébellion morisque qu'il fait à don Juan d'Autriche, Don Juan de Mendoza utilise lui aussi les différentes métaphores du paysage montueux pour souligner la puissance de la révolte qui a pour théâtre la montagne des Alpujarras :

$$
\begin{aligned}
& \text { [...] no hay } \\
& \text { montaña de todas esas } \\
& \text { que no sea parda nube, } \\
& \text { que pardo volcán no sea, } \\
& \text { que de peñascos preñado } \\
& \text { o los reviente o los llueva. }{ }^{41}
\end{aligned}
$$

L'image traditionnelle de la grossesse de la montagne et du volcan est remotivée en une métaphore de l'explosion guerrière. Il s'agit d'une poétique paysagère déjà convenue de la puissance des combats qui sappuie dans cette citation sur une allitération en -p- qui mime l'explosion et une paronomase qui associe le signifiant du rocher, "peñasco ", à celui de la grossesse, "preñado ".

Pourtant, ce n'est pas un paysage champêtre qui nourrit l'anamorphose dans la vision du siège de Galera, mais bien un paysage maritime. Calderón métamorphose la géographie montueuse des Alpujarras en une mer où trois villages choisis en raison de la toponymie illustrent une géographie nautique dès le premier acte dans la bouche de Malec :

$$
\begin{aligned}
& \text { la Alpujarra (aquesa sierra } \\
& \text { que al sol la cerviz levanta, } \\
& \text { y que poblada de villas } \\
& \text { es mar de peñas y plantas, } \\
& \text { adonde sus poblaciones, } \\
& \text { ondas navegan de plata, } \\
& \text { por quien nombres las pusieron } \\
& \text { de Galera, Berja y Gabia). }{ }^{42}
\end{aligned}
$$

Brent De Vos ${ }^{43}$ analyse justement la façon dont Calderón altère la réalité géographique des Alpujarras à des fins poétiques et symboliques. Dans cette métaphore maritime qui structure le paysage militaire des Alpujarras, Calderón choisit des villes très éloignées en réalité en resserrant les liens linguistiques

41. Amar después de la muerte, op. cit., v. 1153-1158.

42. Ibid., v. 180-187.

43. Brent W. De Vos, "Un aspecto de la técnica dramática de Calderón : la geografía y toponimia en El Tuzani de las Alpujarras ", Tinta virtual : revista de estudios hispanos, 1, 2005, pp. 27-34, sur site : http://aix1.uottawa.ca/-gbara083/pages/TintaVirtual01/1\%20JornadasBrent.pdf 
qui rapprochent ces trois villes. "Berja " renvoie à "Verga" que le Diccionario de Autoridades définit dans le lexique nautique comme "las varas o palos de las entenas ", tandis que "galera " et "gavia " appartiennent objectivement au lexique nautique. Se dessine alors une métaphore maritime qui est bientôt appliquée au siège de Galera, dont le site s'apparente à la forme d'une galère, ceci déjà dans les chroniques de Mármol Carvajal et Pérez de Hita d'après Brent De Vos. Face à don Juan d'Autriche, Mendoza décrit le paysage militaire du siège de Galera dans une vision panoramique :

$\quad[. .$.$] Galera es ésta,$
a quien este nombre dieron
o porque su fundación
es así, o ya porque vemos
que a piélagos de peñascos
ondas de flores, batiendo,
sujeta al viento, parece
que se mueve con el viento. ${ }^{44}$

La fonction symbolique d'une telle description évoque déjà le naufrage possible de la cité morisque puisque l'élément dominant est le vent sous l'emprise duquel la ville est placée. Il s'agit d'un jeu conceptiste qui associe le toponyme réel et le nom commun maritime " galera». La métaphore maritime est du reste amplement reprise dans la bouche de don Juan d'Autriche pour exalter la figure militaire d'universelle domination de l'espace que représente ce dernier :

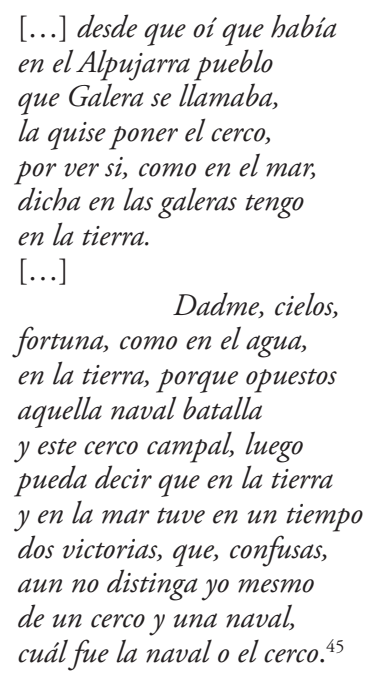

La poétique de l'anamorphose associe le siège terrestre de Galera à une bataille navale. Le jeu conceptiste fondé sur le toponyme Galera repose sur l'utilisation des déictiques qui associent la proximité du spectacle du siège 
terrestre, "este cerco campal ", à la perspective ouverte sur l'espace maritime, " aquella naval batalla ». Le jeu conceptiste permet la duplication de la victoire et le rapprochement historique avec la bataille marine de Lépante comme référence à la gloire essentielle de Don Juan d'Autriche. Brent De Vos commente l'anachronisme historique que Calderón introduit dans la pièce en faisant de don Juan d'Autriche le héros de la bataille maritime de Lépante qui est pourtant postérieure au siège de Galera. La métaphore maritime sert symboliquement à souligner l'idéologie de domination spatiale par les Espagnols en rachetant, comme le souligne Erik Coenen, la violence de Don Juan D'Autriche dans la répression contre les morisques par la mise en relation avec un haut-fait militaire beaucoup plus glorieux ${ }^{46}$.

Cependant, l'association du siège de Galera à la bataille navale de Lépante reste implicite dans le texte caldéronien laissant ouverte l'interprétation de son symbolisme. Au-delà d'un rapprochement historique rétrospectif néanmoins évident avec Lépante, la distorsion topographique et le jeu conceptiste de Amar después de la muerte n'obéit pas seulement à la nécessité poétique d'offrir un espace dramatique frappant par son symbolisme maritime. La poétique de la confusion paysagère et le concepto maritime nourrissent une esthétique du spectacle de la guerre qui évoque aussi la symbolique du naufrage-avecspectateur qui a eu une belle destinée philosophique depuis Lucrèce selon Hans Blumenberg ${ }^{47}$. Comme j'ai pu le remarquer dans mes recherches de thèse à propos de la représentation du naufrage-avec-spectateur ${ }^{48}$, la comedia caldéronienne semble être le témoignage privilégié de l'utilisation qui est faite $\mathrm{du}$ topos issu du "suave mari magno ", lequel ouvre l'éloge de la philosophie en incipit du livre II du De rerum natura de Lucrèce. Il s'avère justement que la conquête meurtrière de Galera par les troupes de don Juan d'Autriche s'apparente pour don Álvaro le Tuzaní des Alpujarras à un naufrage terrestre dont il est le spectateur éploré puisque sa bien-aimée y succombe :

Galera, a quien no en balde
dieron este nombre, ya
zozobrando sobre mares
de purpura que la anegan,
de llamas que la combaten,
se va a pique despeñada
desde esta cumbre a ese valle. ${ }^{49}$

Ce naufrage terrestre illustre la fortune dramatique qui caractérise les Morisques dans ce drame où Calderón semble dénoncer la tragédie historique. Dans cette contemplation par le héros tragique du spectacle du naufrage terrestre, les deux éléments terrestre et maritime, le rocher-galère et la mer-

46. Voir E. Coenen, art. cité, p. 37.

47. Hans Blumenberg, Naufrage avec spectateur, Paris, L'Arche, 1994.

48. Voir, au sujet du naufrage-avec-spectateur, Poétique des espaces naturels..., op. cit., pp. 338-356.

49. El Tuzani de la Alpujarra, v. 2340-2347. 
vallée, sont imbriqués selon la poétique confusionnelle du jeu conceptiste. Ce sont justement les deux mêmes éléments terrestre et maritime que l'on retrouve dans la topographie métaphorique du naufrage-avec-spectateur dans l'incipit à l'éloge de la philosophie. Lucrèce associait la contemplation du naufrage par un spectateur en retrait, depuis la terre ferme, à la contemplation distanciée du panorama de guerre et à une louange du retrait philosophique :

Il est doux, quand sur la vaste mer les vents soulèvent les flots, d'assister de la terre aux rudes épreuves d'autrui : non que la souffrance de personne nous soit un plaisir si grand ; mais voir à quels maux on échappe soi-même est chose douce. Il est doux encore de regarder les grandes batailles de la guerre, rangées parmi les plaines, sans prendre sa part du danger. Mais rien n'est plus doux que d'occuper solidement les hauts lieux fortifiés par la science des sages, régions sereines d'où l'on peut abaisser ses regards sur les autres hommes, les voir errer de toutes parts, et chercher au hasard le chemin de la vie, rivaliser de génie, se disputer la gloire de la naissance, nuit et jour s'efforcer, par un labeur sans égal, de s'élever au comble des richesses ou de s'emparer du pouvoir ${ }^{50}$.

D'après Frank Lestringant, qui commente son utilisation notamment par Montaigne et D’Aubigné, depuis la Renaissance, ce topos est devenu, entre autres, l'emblème du spectacle tragique et de la mise en cause de la position distanciée du spectateur par le truchement du topos du Theatrum mundi:

Le naufragium est l'illustration la plus frappante et la plus extrême du Theatrum mundi, et c'en est aussi le condensé, et comme l'emblème. D'où la combinaison fréquente des deux motifs [...]. La tragédie et le naufrage contemplé depuis la rive ont en commun la place du spectateur, regardant à distance et à l'abri du danger. L'éloignement et le confort du témoin tendent à déréaliser le malheur, et à faire du triste tableau de l'humaine condition une fiction fascinante.

[...] le « suave mari magno» change fondamentalement de sens au crépuscule de la Renaissance, cessant de définir l'idéal de sérénité du sage détaché des convoitises du monde pour représenter une attitude de secrète ironie sur la terre et sur les hommes. [...] Il y entre un malin plaisir à contempler le spectacle des malheurs d'autrui. Cette pointe de volupté maligne qui se glisse désormais dans le motif épicurien est un des plus sûrs indices de la crise de l'humanisme moderne.

Sommes-nous à distance ou déjà dans la barque qui périt ? Peut-on rire de la catastrophe qui nous engloutit, ou qui est bien près de survenir ? Ce qui se dessine à l'époque de la crise de la Renaissance, c'est peut-être, avec cette conscience nouvelle de l'instabilité générale, l'ébranlement du cadre et l'effacement de la frontière impalpable et invisible, mais jusque-là indiscutée, qui séparait le spectateur du spectacle tragique. ${ }^{51}$

Par son association au Theatrum mundi, le sens profond et serein de la métaphore épicurienne de Lucrèce qui introduisait la nécessité du retrait philosophique s'est changé en une interrogation esthétique et éthique sur la "maligne volupté " (selon les termes de Montaigne) que procure le spectacle du malheur d'autrui. De la même façon, à l'aube de l'âge baroque, selon un miroir

50. Lucrèce, De la nature, I, traduction d'Alfred Ernout, Paris, Les Belles Lettres, 1959, Livre II, v. 1-13, p. 71.

51. Frank Lestringant, "Lucrèce, la Renaissance et ses naufrages : à propos du « suave mari magno... ", La renaissance de Lucrèce, Paris, Presses Universitaires de Paris-Sorbonne, 2010, pp. 7-15 (la cit., pp. 13-14). 
de perspectives inversées où l'homme est au centre d'une remise en cause de sa position de spectateur, la comedia caldéronienne fait une exploitation théâtrale inversée du motif épicurien du naufrage-avec-spectateur associé par Lucrèce lui-même à la contemplation distanciée des désastres de la passion guerrière. La métaphore du naufrage militaire dans Amar después de la muerte n'interroge-telle pas la conscience critique du spectateur du théâtre de guerre face au premier spectateur intérieur au massacre qu'est don Álvaro ? Cette métaphore du naufrage avec ce spectateur morisque privilégié, parce que touché par la guerre au plus fort de son amour, n'illustre-t-elle pas la conscience réflexive vis-à-vis des violences de la guerre et du pouvoir que certains critiques ont décelée dans ce drame pseudo-historique ? Le spectateur n'est plus épargné à distance mais bien investi d'une fonction de distanciation critique depuis l'intérieur même du spectacle tragique. Symbole par excellence de la position illusoirement distanciée du spectateur dans les exploitations théâtrales du topos, le rocher devient galère en proie au naufrage, plongeant et engageant le spectateur aux côtés des Morisques dans les affres de l'identification tragique. Ainsi le jeu conceptiste qui permet la confusion des éléments terrestre et maritime illustret-il l'évolution du topos du "suave mari magno".

La position de spectateur impliqué par le spectacle tragique qu'est don Alvaro est préparée symboliquement par doña Clara et doña Isabel qui projettent le paysage de guerre en hors-scène depuis une position provisoirement souveraine issue de l'imaginaire lucrécien du spectateur terrestre du "suave mari magno " :

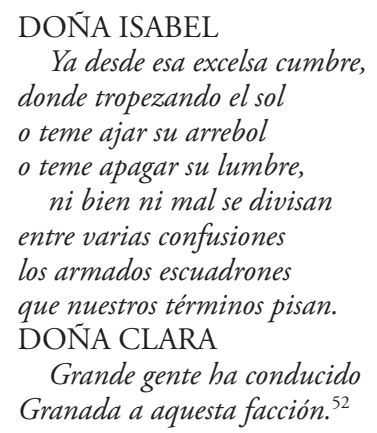

Cette scène intervient alors que doña Clara Maleca vient de s'unir à don Álvaro El Tuzaní. Elle correspond encore à cet instant où le bonheur nuptial est souverain comme transition avant la séparation imminente des mariés.

\section{LA SYMBOLIQUE THÉÂTRALE DES TÉICHOSCOPIES}

Les implications esthétiques du naufrage-avec-spectateur caractérisent indirectement toute une série de spectateurs qui déambulent sur les murailles des comedias caldéroniennes en quête du spectacle de la guerre, telles Laura et Flora, en fonction de spectatrices retranchées dans El sitio de Bredá: 


\author{
LAURA \\ Llégate a ver el campo numeroso, \\ que es a los ojos un objeto hermoso \\ que suspende y divierte \\ FLORA \\ Nuestra ruina en su rigor se advierte. ${ }^{53}$
}

La métaphore spectatoriale est du reste rendue explicite par les comparaisons proférées par le soldat Alonso depuis le camp espagnol dans une scène simultanée, le jeu des scènes parallèles permettant de représenter systématiquement et de façon concomitante les deux camps ennemis :
Bien parece, guardando sus decoros, terrado de Madrid en día de toros, pues verás, si la vista allá enderezas, un alto promontorio de cabezas. ${ }^{54}$

La même situation où Flora et Laura apparaissent à la muraille pour contempler le spectacle du siège se représente à l'acte II, bien qu’à des fins galantes :

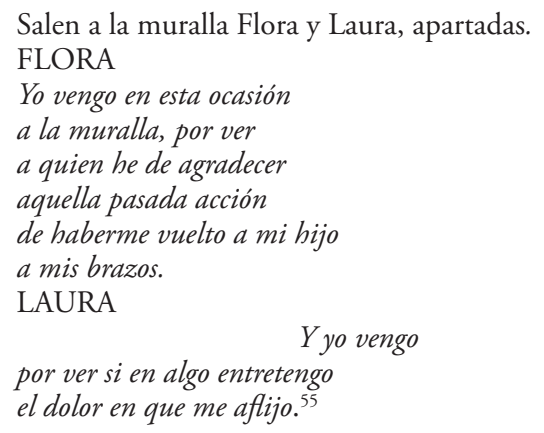

La muraille est l'élément principal autour duquel est structurée la mise en scène de cette comedia, comme le remarque Teresa J. Kirschner ${ }^{56}$. Cependant, au-delà de la muraille comme point de conjonction entre l'intrigue galante et l'intrigue militaire, ces véritables téichoscopies ou "regards depuis la muraille ", si répandus chez Calderón d'après Santiago Fernández Mosquera ${ }^{57}$, illustrent paradoxalement le plaisir procuré par la vision du panorama militaire, « objeto hermoso que suspende y divierte ", dans un écho apparemment superficiel au "suave mari magno » de Lucrèce. Cette position distanciée est représentée dans le corral par " lo alto " ainsi qu'indique la didascalie, c'est-à-dire la première

53. El sitio de Bredá, op. cit., p. 983.

54. Ibid., p. 983.

55. Ibid., p. 1008.

56. Teresa J. Kirschner, «La puesta en escena de El sitio de Bredá : muro y muralla, sitiadores y sitiados ", Ayer y hoy de Calderón, op. cit., pp. 277-289

57. Santiago Fernández Mosquera, "Sobre la funcionalidad del relato ticoscópico en Calderón ", Ayer y hoy de Calderón, op. cit., pp. 259-275. 
galerie $^{58}$. Cette contemplation est si paradoxale pour des assiégées qu'il semble évident que cette introduction d'un spectacle guerrier dans le spectacle théâtral est une évocation d'un théâtre de la guerre comme image du theatrum mundi. Les émotions contrastées de Flora et Laura illustreraient alors, selon une évolution de la métaphore lucrétienne, la double composante émotionnelle face à la tragédie de la guerre qui doit animer le spectateur véritable : plaisir issu de l'identification et horreur tragique. Flora explicite à l'acte III, dans une réflexion sur le spectacle des désastres de la guerre, l'ambivalence de sa situation en tant que spectatrice intérieure de la fureur guerrière :

[...] tanto la voluntad se ve rendida
al hambriento furor, al golpe fuerte,
que duda entre las luces de la vida,
que ignora entre las sombras de la muerte
si asiste el alma a su porción unida,
si falta desasida, y desta suerte,
como a un tiempo dolor y horror recibe
ignora cuándo muere y cuándo vive.

La réaction ambivalente de Flora face au spectacle des désastres de la guerre est une illustration de la remise en cause du détachement du spectateur de la pièce tragique. Dans la tradition lancée par le naufrage-avec-spectateur lucrétien, la position de spectatrice intérieure de Flora tout au long de la pièce est un questionnement tragique de la théorie épicurienne qui veut que l'âme entre en concordance avec les désirs pacifiques du corps en ignorant les élans passionnels qui l'assaillent. Telle est l'analyse que fait Ángel Jacinto Traver Vera du topos du "suave mari magno " pour l'étude qu'il en propose dans la poésie de Fray Luis de León, en reliant justement l'état épicurien d'ataraxie à sa métaphore privilégiée, issue de l'imaginaire maritime :

Epicuro creía que la felicidad humana estribaba en la armonía entre el cuerpo y el alma. El primero reclama calmar la sed, refrescarse ante el calor, saciar el hambre o satisfacer el apetito sexual, necesidades elementales que la naturaleza prodiga por doquier; el espiritu, en cambio, pretende colmar deseos más ambiciosos, como la sed de poder, de dinero, de fama o de inmortalidad. Para los epicúreos sólo si el hombre complacia las exigencias corporales más

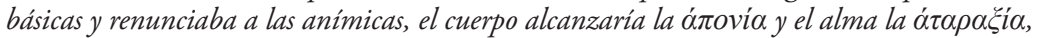
un estado personal de beatitud permanente. [...]

Asi pues, en los escritos epicureos el repertorio visual que pintaba esa paz interior, esa equilibrada dicha, recreaba placenteras escenas naturales, como correlato objetivo entre el concepto y el retrato. Por eso, entre las imágenes preferidas estaban el mar tranquilo, el puerto seguro o, como reverso paralelo, la tormenta marina, que denotaban fácilmente la serenidad, la imperturbabilidad o, viceversa, la discordia del fuero interno. ${ }^{60}$

Juste après la métaphore inaugurale du "suave mari magno ", Lucrèce qualifie justement de ténèbres l'errance passionnelle des hommes en proie au spectacle de leurs propres passions, notamment celui de la guerre qui, en tant

58. Pour la représentation de la muraille dans le corral, voir Teresa J. Kirschner, Art. cité.

59. El sitio de Bredá, op. cit., p. 1023.

60. Ángel Jacinto Traver Vera, « Dos ejemplos de recepción clásica : Lucrecio, 2, 1-13 en Fray Luis y en Lord Byron ", Anuario de estudios filológicos, vol. 22, 1999, pp. 459-474 (la cit., p. 466). 
que passion de l'âme, ne peut assouvir l'aspiration corporelle à l'unité grâce à l'ataraxie :

O misérables esprits des hommes, ô cœurs aveugles! Dans quelles ténèbres et dans quels dangers s'écoule ce peu d'instant qu'est la vie! Ne voyez-vous pas ce que crie la nature ? Réclame-t-elle autre chose que pour le corps l'absence de douleur, et pour l'esprit un sentiment de bien-être, dépourvu d'inquiétude et de crainte ?

Ainsi pour le corps, nous le voyons, il est besoin de bien peu de choses. Tout ce qui peut supprimer la douleur est capable également de lui procurer maint plaisir exquis. Et dans cet état, la nature elle-même ne réclame rien de plus agréable [...].

Aussi puisque pour notre corps les trésors ne sont d'aucun secours, ni la noblesse, ni la gloire du trône, pour le reste, on doit penser qu'ils ne sont pas plus utiles à l'esprit. Est-ce que par hasard, en voyant tes légions pleines d'ardeur se déployer dans le Champ de Mars [littéralement "loca campi ", l'étendue de la plaine ${ }^{61}$ ] et donner l'image de la guerre, soutenue par de nombreuses réserves $<$ par une puissante cavalerie>, pourvues dans chaque camp des <mêmes $>$ armes et animées d'un même courage <en voyant la flotte s'agiter fiévreusement et se déployer au large>, dis-moi, est-ce qu'à ce spectacle les superstitions effrayées s'enfuient tremblantes de ton esprit; est-ce qu'alors les affres de la mort quittent ton cour, le laissant libre et dégagé de souci ? Mais si nous ne voyons là qu'une hypothèse absurde et ridicule, si en réalité les craintes des hommes, les soucis obsédants ne craignent ni le bruit des armes, ni les traits cruels; s'ils hantent audacieusement les rois et les puissants du monde ; s'ils ne respectent ni l'éclat de l'or, ni la brillante splendeur d'un vêtement de pourpre : pourquoi douter que seule la philosophie ait le pouvoir de les mettre en fuite ? [...] Ces terreurs, ces ténèbres de l'esprit il faut donc que les dissipent, non les rayons du soleil ni les traits lumineux du jour, mais l'examen de la nature et son explication. ${ }^{62}$

Calderón adapte cet éloge de la philosophie et la métaphore lucrétienne des terreurs confusionnelles de l'âme en proie au spectacle de la guerre à la réception théâtrale à travers la confusion, exprimée par Flora, entre un état spectatorial d'ataraxie ou de bonheur dans l'impassibilité (" asiste el alma a su porción unida ») et son contraire, la discorde intérieure (" falta desasida ») que Flora traduit hyperboliquement par la métaphore de l'aliénation létale suscitée par la douleur face au spectacle tragique. Calderón formule ainsi la remise en cause du détachement du spectateur propre à l'évolution du topos $\mathrm{du}$ "suave mari magno " par le truchement de son dérivé, la contemplation du spectacle des ravages de la guerre. À travers les deux spectatrices intérieures au théâtre que sont Flora et Laura, dont les noms, liés par une homéotéleute, disent déjà qu'elles représentent les deux composantes dédoublées d'une même attitude du spectateur, la contemplation du spectacle passionnel de la guerre illustre l'ambivalence de la posture du spectateur baroque par rapport à l'état d'ataraxie épicurienne métaphorisée par le "suave mari magno". Dans cette réplique typique de l'état de confusion tragique chez Calderón, vie et mort s'interpénètrent dans l'expression hyperbolique d'une confusion existentielle que ni les lumières du jour ni les ténèbres de la nuit ne dissipent selon une métaphore du clair-obscur émotionnel proche de celui de Lucrèce. Flora y

61. Voir traduction proposée par Charles Guittard dans Lucrèce, De la nature, Paris, Imprimerie Nationale, 2000, p. 115.

62. Lucrèce, $o p$. cit., Les Belles Lettres, v. 14-23, 37-49 et 59-61 
traduit l'horreur du siège de Bréda en invitant le spectateur à la rejoindre dans l'immersion paradoxale qui caractérise désormais le spectateur baroque face au spectacle théâtral, où la sereine ataraxie épicurienne est remplacée par la double composante confusionnelle d'identification et de distanciation théâtrales issue du double sentiment de pitié et d'horreur de la catharsis tragique. La catharsis tragique semble apparaitre dans cette évolution du topos du "suave mari magno" comme un relais de la philosophie naturelle lucrétienne, voie de la démystification de l'humanité.

Dans Amar después de la muerte, l'application de la téichoscopie héritée du "suave mari magno " de Lucrèce à la réception de l'œuvre théâtrale offre, dans le cadre de la diffraction de l'instance spectatoriale, un nouveau développement métaphorique significatif. Dans le récit que le bourreau Garcés fait du meurtre de la jeune femme à don Álvaro, Clara Malec se fait spectatrice de son propre destin en tentant de se retrancher derrière les rideaux de son lit, alors qu'elle est désormais immergée dans le bain de sang du massacre de Galera et sur le point d'être tuée par Garcés. Les rideaux sont alors associés par une comparaison en chiasme, aux pans de muraille d'où symboliquement elle contemplait les factions chrétiennes à l'acte II :

GARCÉS
[...]
Confusa, al fin, y turbada
de verme, como si fueran
las cortinas de una cama
de una muralla cortinas,
detrás se esconde y ampara...
pero con llanto en los ojos
y sin color en la cara
os habéis quedado.
DON ÁLVARO Son
memorias de mis desgracias,
muy parecidas a éstas.

L'inversion de la position distanciée de l'épicurisme lucrétien est traduite par cette métaphore confusionnelle de la muraille depuis laquelle la condamnée à mort, " confusa ", contemple paradoxalement le spectacle tragique du soldat vengeur et de la guerre qui l'investit à jamais. Le détail de la métaphore du rideau, favorisée par le sémantisme figuré de "cortina ", rappelle le rideau théâtral (préférentiellement telón en espagnol) derrière lequel se trouve l'espace des révélations ou également le rideau de toile peinte, "lienzo de murallas " qui, selon Teresa Kirschner et d'après José María Ruano de la Haza ${ }^{64}$, représente souvent la muraille. Dans le récit de Garcés, cette métaphore d'origine téichoscopique correspond du reste significativement, du point de vue du spectateur intérieur don Álvaro, à l'instant de la révélation et de l'identification

63. Amar después de la muerte, op. cit., v. 3021-3030.

64. José María Ruano de la Haza y John J. Allen, Los teatros comerciales del Siglo XVII y la escenificación de la comedia, Madrid, Castalia, 1994, p. 439. 
tragique : le héros embrasse alors, avec la complicité du spectateur véritable de la pièce, le reflet de son propre destin et affronte son désir de vengeance vis-à-vis du meurtrier de sa bien-aimée, qui lui fait, terrible ironie dramatique, le récit de son crime et constate son blêmissement. La réplique de don Álvaro ( «son / memorias de mis desgracias, / muy parecidas a éstas ») pourrait être celle d'un spectateur en pleine identification tragique. La métaphore téichoscopique rejoint alors le topos du "suave mari magno " revu à l'aune de la conscience spectatoriale baroque d'une identification théâtrale, introduite par le spectateur intérieur investi dans le naufrage de la guerre au moment de l'anagnorisis tragique.

Comme le souligne Erik Coenen, Amar después de la muerte offre un exemplum négatif des ravages d'une guerre trop violente, contrairement à $E l$ sitio de Bredá qui présente un exemple positif de l'art du siège. Dans la même optique, Jules Whicker détache tous les éléments d'éthique stoïcienne de la stratégie militaire du marquis de Spinola ${ }^{65}$. Le propos pourrait être nuancé ou davantage envisagé sous l'angle de l'esthétique théâtrale, en insistant sur la véritable nature de l'exemplarité de ces œuvres qui offrent, à partir de l'exemplum militaire, par la diffraction des instances spectatoriales intérieures au drame, une réflexion esthétique sur la réception du spectacle tragique par excellence qu'est la guerre. Les deux pièces nourrissent en effet les deux volets positif et négatif d'une image universelle de la guerre comme spectacle à travers la métaphore du theatrum mundi. Ainsi Don Álvaro utilise-t-il, peu avant le récit de Garcés, au début de l'acte II, la métaphore du théâtre du monde au moment même où il embrasse la conscience du spectacle tragique dans les bras de sa bien-aimée Clara agonisante :

\section{¡Oh montaña inexpugnable de la Alpujarra, ob teatro de la hazaña más cobarde, de la victoria más torpe, de la gloria más infame ${ }^{166}$}

Le rocher inexpugnable, métaphore de la situation épargnée du spectateur lucrétien, devient l'espace tragique par excellence de l'investissement du spectateur par le spectacle tragique. Noyé au cœur du paysage militaire et investi par sa violence, le personnage de ce théâtre de guerre devient une conscience réflexive engagée, messagère des ravages de la guerre, invitant le spectateur véritable à la catharsis.

Calderón est fidèle à la vision lucrétienne de la guerre, telle que Lucrèce l'a héritée de l'épicurisme. La dévorante violence guerrière est rejetée par Lucrèce

65. Jules Whicker, «"la caballería bajo fuego": la representación de la virtud militar española en El asalto de Mastrique de Lope y El sitio de Bredá de Calderón ", Calderón 2000, II, Kassel, Reichenberger, 2002, pp. 411-423.

66. Ibid., v. 2773- 2777. 
comme emblème du désordre induit par les passions de l'âme, comme le remarque Jean Salem dans une introduction à l'épicurisme de Lucrèce et au topos $\mathrm{du}$ "suave mari magno":

La guerre : Épicure lui-même semble l'avoir considérée comme un terrible désordre et comme la pire des conjonctures parmi toutes celles auxquelles le sage doit apprendre à se préparer. L'épicurien est foncièrement un pacifiste : Lucrèce le laisse entendre encore plus nettement qu'Épicure. La guerre, semble nous dire le poème De la nature, n'est rien qu'une affaire de rapine et de désirs morbides. [...] il y a chez Lucrèce, une horreur absolue de la guerre, du sacrifice, de la souffrance. C'est que la guerre, si elle permet à l'épicurien de fournir une image commode de la réalité physique, constitue en réalité l'antithèse la plus dévastatrice de la vie, de la sagesse et du plaisir. ${ }^{67}$

Laissons les métaphores mêmes de Lucrèce suggérer combien la guerre est l'emblème ultime de la vaine et passionnelle conquête matérielle que l'homme prend comme un succédané du bonheur véritable qui est dans l'ataraxie :

Ainsi donc le genre humain travaille sans profit, en pure perte, toujours, et se consume dans de vains soucis : évidemment c'est qu'il ne connaît pas la limite de la possession, et jusqu'où peut s'étendre le véritable plaisir. Et cette ignorance peu à peu nous a entraînés dans la tempête, et a déchaîné les orages et les ruines de la guerre. ${ }^{68}$

Comme antithèse à l'immersion spectatoriale paradoxale de Flora dans El sitio de Bredá et de don Álvaro dans Amar después de la muerte, Calderón nous offre à travers la menace de guerre sanguinaire de Fierabrás, à l'acte I de $L a$ puente de Mantible, une caricature de la guerre comme emblème d'une attitude passionnelle destructrice. Et pour ce faire, il place à la fin de la pièce le géant Fierabrás en position de théicoscopique en haut du pont qui surplombe le combat entre les armées françaises et les armées africaines auxquelles le géant préside. De sa position faussement distanciée, Fierabrás, le mauvais spectateur, éprouve une fière jouissance passionnelle, condamnable du point de vue épicurien :

\author{
Los sitiados de mi tierra, \\ viendo que ya se corona \\ el Mantible de pendones \\ que la lis de Francia borda, \\ se han atrevido a salir \\ $y$, marchando en buena forma, \\ se van acercando al puente; \\ los franceses, que blasonan \\ de que los han de librar, \\ osados las armas toman. \\ $Y$ en medio de todos, yo \\ con ufana vanagloria \\ estoy de ver el cuidado \\ que les da una vida sola; \\ [...] \\ Ya por las dos partes llegan \\ divididas las dos tropas;
}

67. Jean Salem, «Lucrèce et l'épicurisme, introduction générale ", La renaissance de Lucrèce, op. cit., pp. 19-34 (la cit., p. 22).

68. Lucrèce, De rerum natura, op. cit., II, livre V, v. 1430-1435. 


\section{bien podré hablar desde aqui, porque dos campos me oigan. [...] Sobre el puente de Mantible os espera mi persona. ${ }^{6}$}

La position provisoirement épargnée de Fierabrás, qui est une imitation déformée du retrait philosophique lucrétien ("con ufana vanagloria / estoy de ver el cuidado / que les da una vida sola »), va bientôt être démentie car la bataille gagne rapidement le pont. Fierabrás subit alors une chute symbolique depuis le pont crénelé représenté par la première galerie du théâtre, ainsi que le remarque Arminda :

\section{$Y$ de la más alta almena}

bárbaro un turco se arroja

hasta llegar a tus pies.

Cae rodando desde lo alto Fierabrás muy sangriento y sin espada. $^{70}$

À travers ce jeu d'inversions spectatoriales, la pièce offre une remise en cause de la position du spectateur épargné par le spectacle de la guerre en restant cependant fidèle à la conception lucrétienne de la guerre et en prévenant une mauvaise interprétation du détachement épicurien comme " maligne volupté " tel qu'il est reformulé à partir de la Renaissance. Par cette posture erronée de Fierabrás comme spectateur intérieur qui se complaît dans la jouissance passionnelle face au spectacle, Calderón offre un message d'éducation à la réception du théâtre tragique. Le spectateur véritable est incité à la catharsis à travers l'exemple d'une passion guerrière qui envahit le spectateur intérieur et le précipite vers sa perte.

Au début du troisième acte de cette même pièce, le valet Guarín, porteparole de la méta-théâtralité en bon valet de comedia qu'il est, ne réclame-t-il pas sa place sur la muraille représentée par la première galerie du corral comme une invitation au spectacle : « $¡ \mathrm{Oh}$, quien boleta tuviese / para algún balcón del cielo / en fiesta que es tan solene ! $\aleph^{71}$. La métathéâtralité tragique de la guerre dans les drames légendaires caldéroniens est le reflet d'un apprivoisement symbolique des conditions limitées de représentation de la guerre dans le corral selon l'influence croisée du topos dérivé du "suave mari magno " de Lucrèce.

De la même façon, une muraille réflexive semblable à celle de El sitio de Bredá offre un point de vue sur le combat en téichoscopie au troisième acte de El principe constante lorsque Cutiño, le valet de don Fernando, contemple de façon souveraine, aux côtés du tombeau de son maître sur la première galerie, la victoire des armées chrétiennes de don Alfonso éclairées par la grâce du martyr don Fernando :

([...] descúbrese en el muro un ataúd cubierto con un paño negro y CUTIÑO a su lado, y salen el REY y ZELÍN por bajo. $)^{72}$

69. La puente de Mantible, op. cit., pp. 569-570.

70. Ibid., p. 571.

71. Ibid., p. 549.

72. El principe constante, op. cit., didascalie qui suit le vers 2764 . 


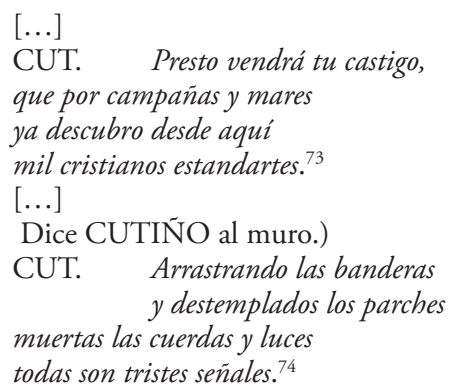

La téichoscopie fait du valet le porte-parole du theatrum mundi de la tragédie guerrière en hors-scène, dépassée par la réalité de la grâce divine. Il s'agit là d'une adaptation métaphysique de la métaphore du retranchement épicurien de Lucrèce. Elle est l'aboutissement de la métaphore théâtrale développée au premier acte autour de la bataille funeste qui conduit Fernando à l'esclavage. Dans un récit téichoscopique de l'acte I, don Juan relate à Fernando l'attaque des armées maures :

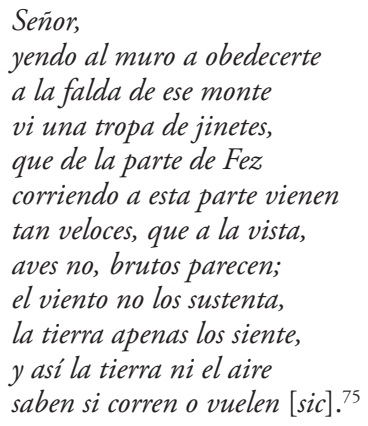

Ce récit trouve son pendant de façon parallèle au troisième acte dans la vision finale que Cutiño a depuis la muraille de l'armée maure mise en déroute par l'armée chrétienne. L'inversion sert la caractérisation de la théâtralité de la guerre comme image emblématique du théâtre du monde. Au premier acte, en effet, la même réversibilité de la victoire introduit une référence directe au theatrum mundi qui éclaire la conception caldéronienne de la guerre. Alors que Fernando, provisoirement victorieux, rejoint le capitaine maure Muley, désarçonné dans le combat qui l'oppose aux chrétiens, il commente la métathéâtralité de la guerre : "En la desierta campaña / que tumba común parece I de alarbes cuerpos, si ya / no es teatro de la muerte ${ }^{76}$. La guerre est l'image d'une fortune changeante. La mise en scène schématique des revirements de fortune des batailles illustre la symbolique de la guerre comme theatrum mundi. Du reste, au terme de ce premier acte, pour clore justement les revirements de la 
funeste bataille, le portugais don Juan s'exclame : " Oh infelice jornada! » ${ }^{77}$. La polysémie du mot " jornada» fait autant référence à l'expédition militaire ("vale también la expedición a que se destina el ejército " indique Le dictionnaire de Autoridades) qu'à la fin de l'acte appelé "jornada", mais elle peut aussi évoquer le destin mystique de don Fernando car la "jornada" désigne également le parcours de l'âme vers Dieu (Autoridades) : "En lo mystico se toma por el tiempo de la vida, y también por el fin de ella : especialemente la que se aprehende que hace el alma, hasta presentarse en el Tribunal de Dios». La métaphore téichoscopique du retranchement face à la guerre de l'existence comme posture épicurienne chez Lucrèce est adaptée à l'ascèse de don Fernando via l'application de la métaphore à l'univers du théâtre. La métathéâtralité de la réplique "infelice jornada " à l'acte I souligne la métaphore de la guerre comme grand théâtre du monde qui nourrit prémonitoirement une vérité métaphysique.

La même métaphore théâtrale liée à la guerre se retrouve dans La vida es sueño. La guerre y est associée à la tragédie. Calderón prête à Basilio une métaphore traditionnelle à propos de la guerre civile, dont les échos pénètrent jusque dans le palais : "El dosel de la jura, reducido / a segunda intención, a honor segundo, I teatro funesto es, donde importuna / representa tragedias la fortuna ${ }^{78}$. Et Estrella de lui faire écho : "todo es desdichas, y tragedias todo $»^{79}$. La métathéâtralité tragique de la guerre réapparaît à l'envi dans toutes les pièces du corpus qui traitent d'une guerre légendaire à l'origine de revirements de fortune ; dans La gran Cenobia: " ha de ser / teatro este monte fuerte, / romanos, de vuestra muerte $"$ " fortuna $»^{81}$. Dans cette pièce, les combats répétées conduisent à des revirements de fortune aussi subits que surprenants, tout comme dans La puente de Mantible où la guerre est sans cesse contemplée comme un spectacle tragique depuis la tour où sont assiégés les Français : "Yo ver puestos frente a frente / dos campos que se amenazan, / representando a los cielos / en teatros de esmeraldas / mil tragedias la fortuna $"{ }^{82}$. La guerre est l'image des vaines gloires mondaines affectées par l'inconstance du sort : elle est le spectacle tragique au-delà duquel la comedia échappe généralement à la tragédie, comme cela se produit à la fin de La vida es sueño avec la catharsis intérieure au drame favorisée par la mort de Clarín. Comme Guarín dans La puente de Mantible, Clarín, dans La vida es sueño, commente la théâtralité de la guerre lorsqu'il se cache dans le monte pour échapper au combat : "Escondido desde aqui / toda la fiesta he de ver ${ }^{83}$. On peut supposer que Clarín se réfugie derrière les roches en carton-pâte qui se trouvent en haut de l'escalier scénique qui représente le monte, d'où il tombe peu après, atteint par une balle perdue, délivrant une morale quant au refus

77. Ibid., v. 970.

78. La vida es sueño, op. cit., v. 2440-2443.

79. Ibid., v. 2467.

80. La gran Cenobia, op. cit., p. 349.

81. Ibid., p. 367.

82. La puente de Mantible, op. cit., p. 508.

83. La vida es sueño, op. cit., v. 3054-3055. 
de s'engager dans le combat. La métaphore théâtrale vient rejoindre toutes les allusions métathéâtrales qui caractérisent le valet au fil de la pièce, mais elle constitue aussi l'apothéose de ce courant métaphorique qui associe le spectacle dans le spectacle qu'est la guerre à un theatrum mundi. Clarín est le biennommé, parce qu'en bon valet de comedia qu'il est, il claironne certes à tort et à travers mais également, en véritable instrument d'une guerre existentielle, il claironne la victoire que peut représenter le combat contre la fatalité s'il est assumé. En tant que clairon, il signe la fin de la tragédie de la guerre par sa mort symbolique et permet à la pièce de dépasser le tragique.

La représentation de la guerre comme un spectacle interne livré à l'imagination du spectateur dans les premières comedias de Calderón cristallise donc une fascination qui prend des valeurs existentielles symboliques. Dans presque toutes les pièces, le spectacle de la guerre est amplement mis en scène à travers les spectateurs intérieurs dont les différentes postures poétiques par rapport au combat illustrent une esthétique de la réception de l'œuvre tragique. Dans Amar después de la muerte, la métaphore du naufrage-avec-spectateur et de la muraille nourrit une vision réflexive sur les désastres de la guerre. Quant à El sitio de Bredá, le regard perpétuel et complaisant des assiégées Flora et Laura en téichoscopie illustre une conscience théâtrale qui est destinée à sublimer la victoire exemplaire des stratégies galantes et militaires des Espagnols. Au-delà des guerres historiques que ces deux pièces mettent en scène, c'est une vision universelle de la guerre comme reflet du spectacle tragique qu'elles suggèrent, en invitant le spectateur à une catharsis confusionnelle qui mêle à la fois la distanciation et l'identification grâce à la représentation de faits historiques relativement proches de la réception de l'époque. On le voit, la théâtralité de la guerre, propre à susciter l'identification du spectateur, pourrait bien être la réponse esthétique et distanciée à une "culture de la guerre " ${ }^{84}$, probablement intimement partagée par le public de l'époque.

Présente dans presque toutes les pièces, la métaphore de la tragédie de la guerre nourrit la réflexion sur la vanité et la réversibilité des gloires mondaines tandis que la diffraction de l'instance spectatoriale délivre une esthétique de l'identification théâtrale. Dans El principe constante, la représentation théâtralisée de la guerre accompagne la symbolique de la fortune inconstante qui caractérise le sort du Prince constant, don Fernando. Quant à La vida es sueño, l'épisode de la mort de Clarín, clairon de la tragédie de la guerre, constitue l'aboutissement des techniques représentatives de la guerre apprivoisées à des fins symboliques. L'épisode est une image de la réflexivité du genre théâtral qui invite à la catharsis, telle qu' elle apparaît en germe dans La puente de Mantible à travers la démultiplication de l'instance spectatoriale : le combat existentiel doit être assumé car, contrairement au retrait philosophique prôné par Lucrèce,

84. Voir David García Hernán, La cultura de la guerra..., op. cit., notamment la conclusion de l'ouvrage, pp. 273-282. 
toute position, même celle du spectateur de la guerre, peut être atteinte par la réversibilité du destin. La métaphore de Lucrèce est adaptée à l'esthétique tragique : nul n'échappe au combat de sa propre existence, chacun peut être «embarqué » dans le naufrage de son destin mais il convient de maintenir une distance réflexive, malgré tout, face à ce spectacle de l'inconstance du sort, du theatrum mundi. La poétique du théâtre de guerre, nourrie d'héritages esthétiques divers, permet l'intégration des préoccupations historiques tout en proposant une vision complexe de la guerre, faite de fascination et de distanciation. Puissamment retravaillée par l'esthétique tragique, la "culture de la guerre» pourrait bien faire du spectateur intérieur l'image du spectateur du Siècle d'Or aux prises avec le destin militaire de sa société. Au niveau de la réception théâtrale, la nécessaire distanciation qui est à l'origine du plaisir du spectacle s'accompagne de l'identification selon laquelle le spectateur doit atteindre à la purgation des différentes passions individuelles dont la guerre est l'emblème universel : fascination ou horreur spectatoriales pour Laura et Flora dans El sitio de Bredá, violence, cupidité et orgueil pour Fierabrás dans $L a$ puente de Mantible, mais aussi douleur de la perte et désir de vengeance pour don Álvaro dans Amar después de la muerte ou encore peur du combat et de l'engagement existentiel pour Clarín dans La vida es sueño. Tel est le message claironné par le théâtre de guerre caldéronien. 